\title{
Cole-Cole Plots for Linear and Nonlinear Dielectric Relaxation in Solutions of Rigid, Highly Dipolar, Symmetric-Top Molecules in Spherical Solvents
}

\author{
W. Alexiewicz* AND K. Grygiel \\ Nonlinear Optics Division, Institute of Physics \\ Adam Mickiewicz University \\ Umultowska 85, 61-614 Poznań, Poland
}

(Received May 9, 2008)

\begin{abstract}
The graphical analysis of the influence of the rotational diffusion tensor anisotropy and the orientation of the permanent dipole moment on the linear and nonlinear dielectric relaxation is shown. The solution of Smoluchowski-Debye rotational diffusion equation for rigid, and noninteracting polar, symmetric-top molecules, in the "weak molecular reorientation approximation", was used. In order to highlight the influence of the symmetric shape of molecule, in comparison with classical, spherical-top Smoluchowski rotational diffusion, we present sets of Argand-type plots and three-dimensional Cole-Cole diagrams for linear and nonlinear electric susceptibilities. The results indicate that, in describing the nonlinear dielectric relaxation, the simplest spherical-top rotational diffusion model may be a sufficient approximation in some special cases only.
\end{abstract}

PACS numbers: 05.40.Jc, 77.22.-d, 77.22.Gm

\section{Introduction}

The aim of this paper is to give graphical analysis of the influence of the symmetric-top shape of the rigid, highly dipolar molecule and the orientation of its permanent dipole moment on the phenomena of the linear and nonlinear dielectric relaxations in dilute solutions, in comparison with these efects for the

*corresponding author; e-mail: walex@amu.edu.pl 
spherical-top molecules. The starting point are the results of our paper [1], being a continuation of the previous theories of Kielich and co-workers [2-7]. We present a set of the Cole-Cole plots for the dielectric relaxation based on an approximate solution of the classical Smoluchowski-Debye rotational diffusion equation.

The Smoluchowski equation for rotational diffusion of molecules plays the important role in linear relaxation phenomena [8], in the induced dynamic optical birefringence [2, 9-13], and in nonlinear - the Langevin type relaxation [2, 3, $14,15]$. An extensive review of the actual progress in the wide theory of rotational diffusion, including the introduction to the models of "anomalous rotational diffusion" - the fractional diffusion extension of the classical Smoluchowski equation — was given by Coffey [16].

The experiments on nonlinear dielectric relaxation effect in liquids have been performed for many years in Leuven and Poznań by De Smet, Hellemans, Jadżyn, Kędziora and co-workers [17-21].

Section 2 presents our previous result [1] for the linear and nonlinear Langevin type electric susceptibilities, which make the basis for the detailed graphical analysis. In Sect. 3 we discuss the changes following from the symmetric shape of molecule in comparison with the model of its spherical shape. These changes may be of importance in many cases, so the spherical-top rotational diffusion model may be a good approximation in some special cases.

\section{Linear and nonlinear electric susceptibilities}

We consider the isotropic dielectric liquid composed of noninteracting, rigid and symmetric-top molecules with the permanent dipole moment equal to $\mu$, acted on by a strong external dc bias electric field $E_{Z}$ superimposed on a weak harmonic electric field $E_{\omega} \cos \omega t$ with the frequency $\omega$ :

$E_{Z}(t)=E_{Z}+E_{\omega} \cos \omega t$.

The electric polarization induced in the medium $\left\langle P_{Z}(t)\right\rangle$ may be written as a sum

$$
\left\langle P_{Z}(t)\right\rangle=\left\langle P_{Z}^{(1)}(t)\right\rangle+\left\langle P_{Z}^{(3)}(t)\right\rangle
$$

of the linear $\left\langle P_{Z}^{(1)}(t)\right\rangle$ and nonlinear $\left\langle P_{Z}^{(3)}(t)\right\rangle$ parts. It is well known that

$$
\left\langle P_{Z}^{(1)}(t)\right\rangle=\varepsilon_{0} \chi^{*}(-\omega ; \omega) E_{\omega}
$$

where $\varepsilon_{0}$ denotes the electric permittivity of the vacuum and $\chi^{*}(-\omega ; \omega)$ is the complex, linear susceptibility of the liquids. The third-order electric polarization depends on the complex nonlinear susceptibility of the liquid $\chi^{*}(-\omega ; \omega, 0,0)$

$$
\left\langle P_{Z}^{(3)}(t)\right\rangle=\varepsilon_{0} \chi^{*}(-\omega ; \omega, 0,0) E_{\omega} E_{Z}^{2} .
$$

Both these susceptibilities may be written in familiar form, as a sum of the real and imaginary parts

$$
\chi^{*}(-\omega ; \omega)=\chi^{\prime}(-\omega ; \omega)-\mathrm{i} \chi^{\prime \prime}(-\omega ; \omega)
$$

and 


$$
\chi^{*}(-\omega ; \omega, 0,0)=\chi^{\prime}(-\omega ; \omega, 0,0)-\mathrm{i} \chi^{\prime \prime}(-\omega ; \omega, 0,0),
$$

where $\mathrm{i}^{2}=-1$. The dependence of susceptibilities (5), (6) of molecular liquids on the frequency, shape of the molecules and orientation of the permanent dipole moment is the central point of the linear and nonlinear relaxation experimental and theoretical investigation.

From the Smoluchowski-Debye rotational diffusion of noninteracting, rigid and symmetric-top molecules, in "the weak molecular reorientation approximation", the following result has been obtained $[1,6,9,10]$ :

$$
\begin{aligned}
& 3 k T \varepsilon_{0} \chi^{\prime}(-\omega ; \omega)=\rho \mu^{2} C_{1}^{\prime}(-\omega ; \omega), \\
& 3 k T \varepsilon_{0} \chi^{\prime \prime}(-\omega ; \omega)=\rho \mu^{2} C_{1}^{\prime \prime}(-\omega ; \omega),
\end{aligned}
$$

where $\rho$ denotes the density of the liquid, $k$ - the Boltzmann constant and $T-$ Kelvin temperature. We see that the real and imaginary parts of the susceptibility $\chi^{*}(-\omega ; \omega)$, depending obviously on the electric field frequency $\omega$, are functions of the parameter of the rotational diffusion tensor anisotropy

$$
\xi=D_{x x} / D_{z z}
$$

and the polar angle $\Theta$ between the permanent dipole moment and the symmetry axis of the molecule. From the Smoluchowski-Debye equation of rotational diffusion of the symmetric-top molecule, we have [1]:

$$
C_{1}^{\prime}(-\omega ; \omega)=s_{11}(\omega, \xi) \sin ^{2} \Theta+s_{10}(\omega) \cos ^{2} \Theta
$$

and

$$
C_{1}^{\prime \prime}(-\omega ; \omega)=-\omega \tau_{11} s_{11}(\omega, \xi) \sin ^{2} \Theta-\omega \tau_{10} s_{10}(\omega) \cos ^{2} \Theta
$$

where

$$
s_{l m}(\omega, \xi)=\left(1+\omega^{2} \tau_{l m}^{2}\right)^{-1}
$$

are well-known Debye-Kielich relaxational functions depending on the molecular relaxations times of the symmetric-top molecules

$$
\tau_{l m}=D_{z z}^{-1}\left[l(l+1)-m^{2}(1-\xi)\right]^{-1} .
$$

These functions and the relaxation times are independent of $\xi$ when $m=0$. We see, Eqs. (8), (9), that for the symmetric-top molecules, the linear dielectric relaxation depends in fact on the superposition of two molecular rotations, characterized by two relaxation times $\tau_{10}$ and $\tau_{11}$, respectively around the long and short molecular axis. Similarly, for the nonlinear dielectric relaxation we have

$$
\begin{aligned}
& 45 k^{3} T^{3} \chi^{\prime}(-\omega ; \omega, 0,0)=\rho \mu^{4} C_{2}^{\prime}(-\omega ; \omega, 0,0), \\
& 45 k^{3} T^{3} \chi^{\prime \prime}(-\omega ; \omega, 0,0)=\rho \mu^{4} C_{2}^{\prime \prime}(-\omega ; \omega, 0,0),
\end{aligned}
$$

the result is valid only in the case of "weak molecular reorientation approximation" and in the absence of polarizability of the molecules. The nonlinear electrical susceptibilities of the molecule in Eqs. (12) are in this case equal to [1]:

$$
C_{2}^{\prime}(-\omega ; \omega, 0,0)=a_{11}^{x x x}(\omega, \xi) \sin ^{4} \Theta
$$




$$
+\left[a_{10}^{x x z}(\omega, \xi)+a_{11}^{x z z}(\omega, \xi)\right] \sin ^{2} \Theta \cos ^{2} \Theta+a_{10}^{z z z}(\omega) \cos ^{4} \Theta
$$

and

$$
\begin{aligned}
C_{2}^{\prime \prime}(-\omega ; \omega, 0,0)=b_{11}^{x x x}(\omega, \xi) \sin ^{4} \Theta \\
\quad+\left[b_{10}^{x x z}(\omega, \xi)+b_{11}^{x z z}(\omega, \xi)\right] \sin ^{2} \Theta \cos ^{2} \Theta+b_{10}^{z z z}(\omega) \cos ^{4} \Theta .
\end{aligned}
$$

The explicit formulae for the dispersion $-a_{10}^{z z z}(\omega), a_{10}^{x x z}(\omega, \xi), a_{11}^{x z z}(\omega, \xi)$, $a_{10}^{z z z}(\omega)$ and absorption $-a_{10}^{z z z}(\omega), b_{10}^{x x z}(\omega, \xi), b_{11}^{x z z}(\omega, \xi)$ and $b_{10}^{z z z}(\omega)$ functions were derived in [1], they are simply some superpositions of the Debye-Kielich factors given by Eq. (11). We see that in the nonlinear relaxation, the dispersion and absorption phenomena, Eqs. (13), (14), can be interpreted as a result of the superpositions of three independent molecular motions:

- the diffusional rotation around the long molecular axis, terms with $a_{10}^{z z z}(\omega)$ or $b_{10}^{z z z}(\omega)$, independent of the parameter $\xi$, and

- the rotation around the short axis, terms with $a_{11}^{x x x}(\omega, \xi)$ or $b_{11}^{x x x}(\omega, \xi)$, respectively,

- and the rotation connected with third term of the "mixed" rotational motion, with the functions $a_{10}^{x x z}(\omega, \xi), a_{11}^{x z z}(\omega, \xi)$ and $b_{10}^{x x z}(\omega, \xi), b_{11}^{x z z}(\omega, \xi)$ multiplied by $\sin ^{2} \Theta \cos ^{2} \Theta$.

\section{Influence of symmetric shape and dipole moment orientation of the molecule on linear and nonlinear Cole-Cole plots}

It is very convenient to analyze the frequency dependence of the rotational relaxation in liquids with the familiar Cole-Cole plots [4] of the imaginary part of the electric susceptibility as a function of its real part. The Cole-Cole plots for the linear susceptibilities $C_{1}^{\prime}(-\omega ; \omega)$ and $C_{1}^{\prime \prime}(-\omega ; \omega)$ given by Eqs. (7), on the angle $\Theta$ between the permanent dipole moment and the symmetry axis of the molecule, for some values of the parameter of the rotational diffusion anisotropy $\xi$, are given in Figs. 1. Although the linear relaxation does not clearly depend, for $\xi<1$, on the anisotropy of the rotational diffusion tensor, in the opposite case, for $\xi>1$, distinct changes are seen. The "anisotropic term", with the relaxational function $s_{11}(\omega, \xi)$, gives the maximal contribution when $\Theta=90^{\circ}$. For dipole moment lying along the molecular $z$-axis, $\Theta=0$, and the Smoluchowski-Debye equation for the spherical-top becomes a very good approximation.

The Cole-Cole plots for the normalized linear susceptibilities $C_{1}^{\prime}(-\omega ; \omega)$ and $C_{1}^{\prime \prime}(-\omega ; \omega)$ given by Eqs. (7), and projected onto the plane $\left(\Theta, C_{1}^{\prime}(-\omega ; \omega)\right)$, for selected values of the parameter of the rotational diffusion anisotropy $\xi$, are given in Fig. 2. We see that for increasing values of $\xi$, distinct changes from the classical Cole-Cole semicircle appear if $\Theta=90^{\circ}$.

The Cole-Cole plots for the susceptibilities $C_{1}^{\prime}(-\omega ; \omega)$ and $C_{1}^{\prime \prime}(-\omega ; \omega)$, given by Eqs. (7), on the parameter of the rotational diffusion anisotropy $\xi$, for some values of the angle $\Theta$ between the permanent dipole moment and the symmetry axis of the molecule, are given in Fig. 3. 

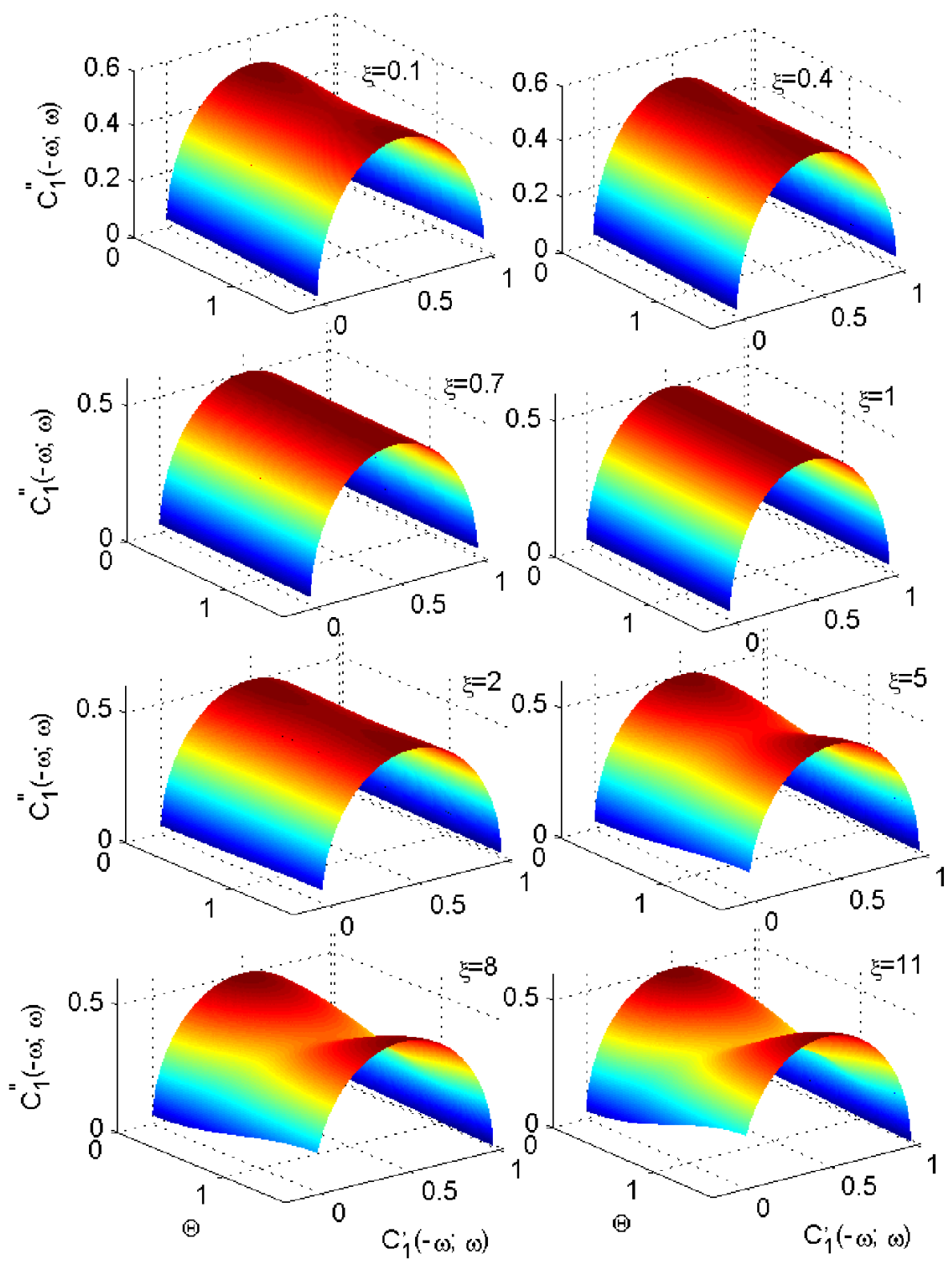

Fig. 1. Dependence of the linear Cole-Cole plots, resulting from Eqs. (7), of the angle $\Theta$ between permanent dipole moment and the symmetry axis of the molecule, for some values of the parameter of the rotational diffusion anisotropy $\xi$. The angle $\Theta$ is changing from 0 to $\pi / 2$. 


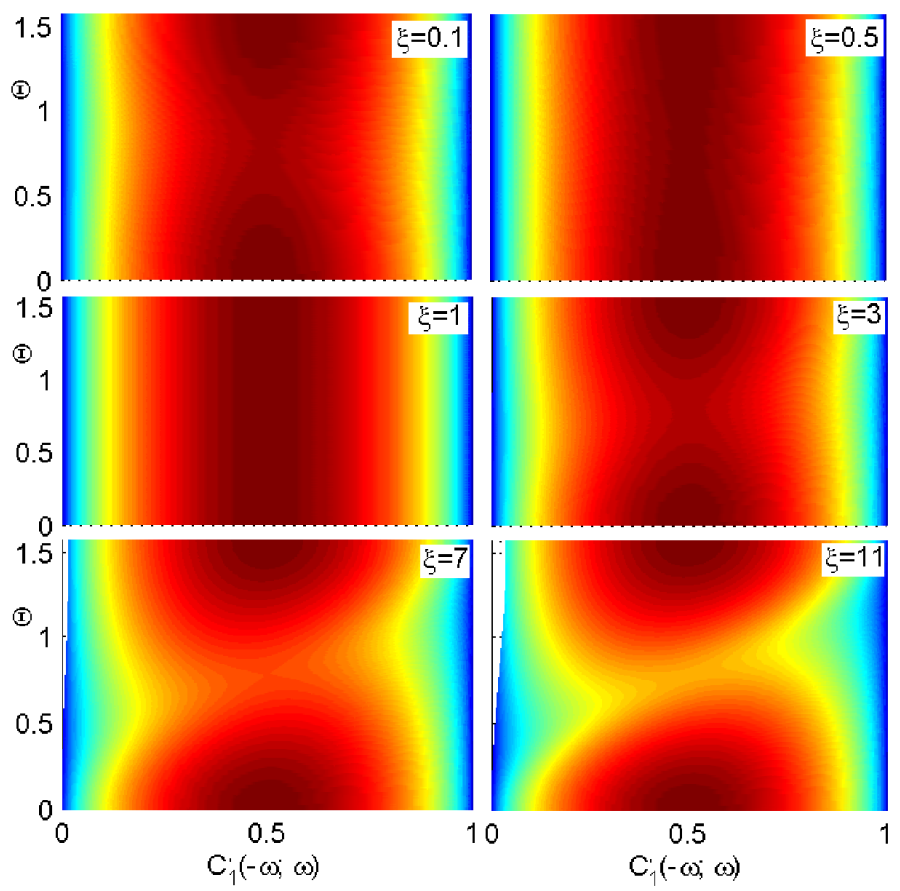

Fig. 2. Cole-Cole plots for the linear susceptibilities $C_{1}^{\prime \prime}\left(C_{1}^{\prime}\right)$, given by Eqs. (7), projected on the plane $\left(\Theta, C_{1}^{\prime}(-\omega ; \omega)\right)$ for some values of the parameter of the rotational diffusion anisotropy $\xi$.

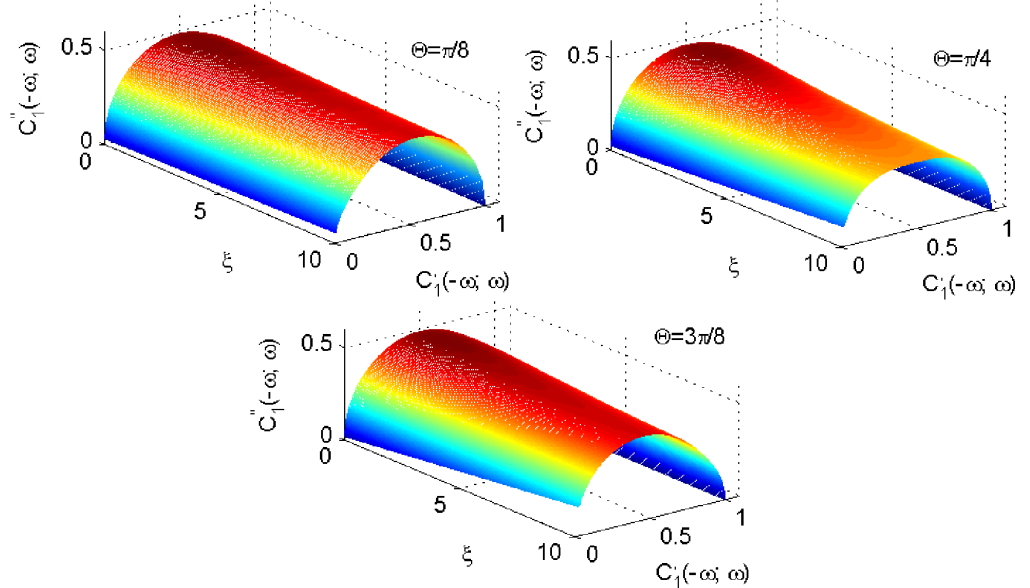

Fig. 3. Dependence of Cole-Cole plots for the linear susceptibilities $C_{1}^{\prime \prime}\left(C_{1}^{\prime}\right)$, given by Eqs. (7), on the parameter of the rotational diffusion anisotropy $\xi$, for some values of the angle $\Theta$ between permanent dipole moment and the symmetry axis of the molecule. 

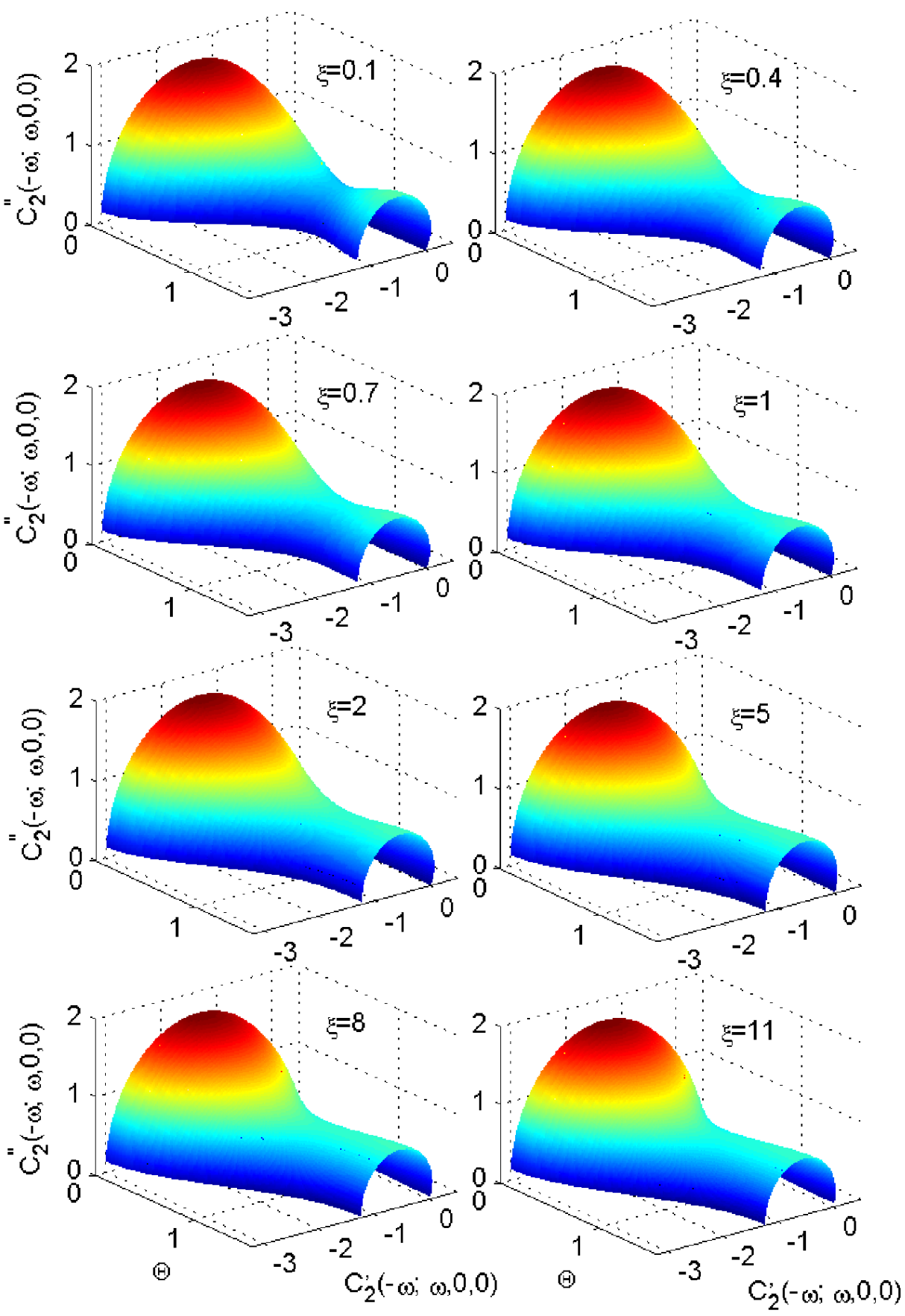

Fig. 4. Dependence of the nonlinear Cole-Cole plots, resulting from Eqs. (12)-(14), of the angle $\Theta$ between permanent dipole moment and the symmetry axis of the molecule for some values of the parameter of the rotational diffusion anisotropy $\xi$. 


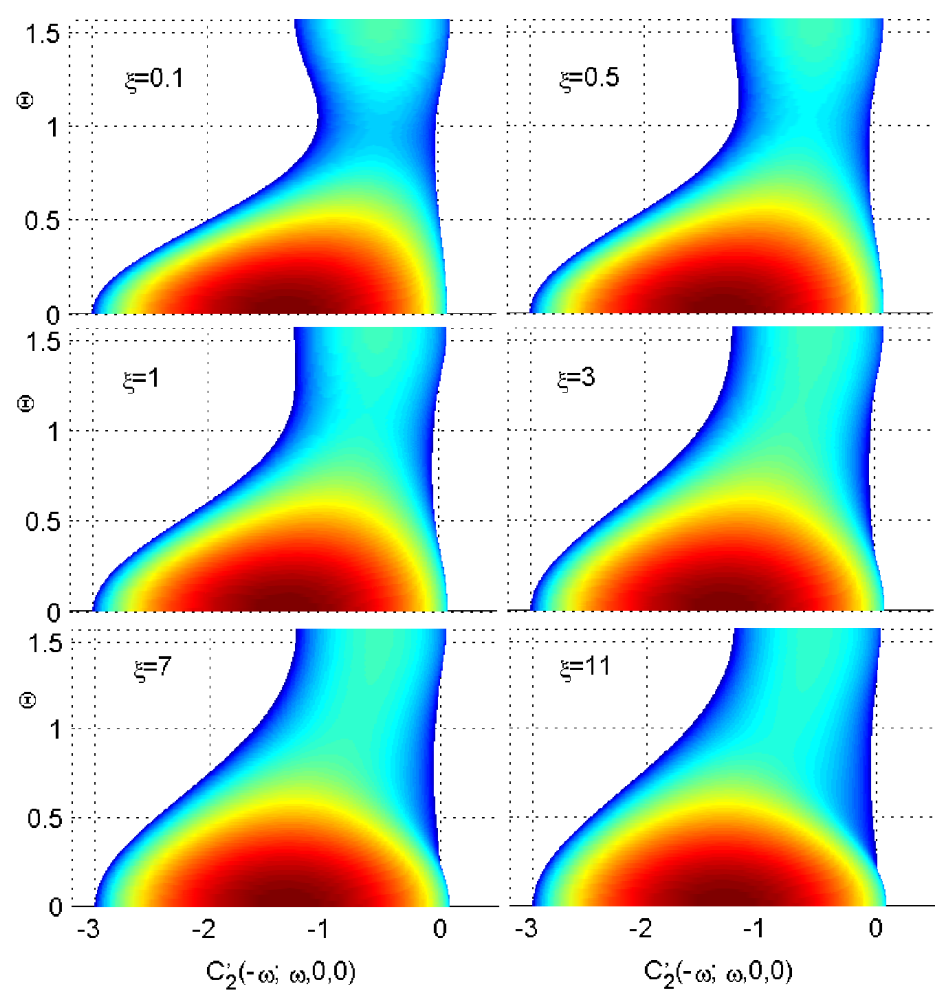

Fig. 5. Cole-Cole plots for the nonlinear susceptibilities $C_{2}^{\prime \prime}\left(C_{2}^{\prime}\right)$, given by Eqs. (12)(14), projected on the plane $\left(\Theta, C_{2}^{\prime}(-\omega ; \omega, 0,0)\right)$ for some values of the parameter $\xi$.

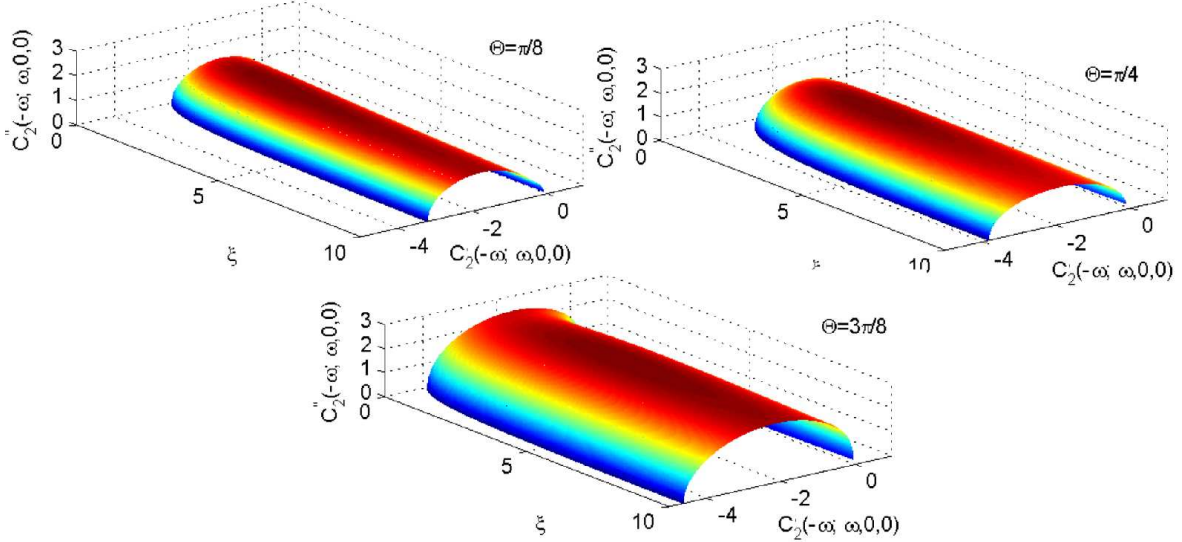

Fig. 6. Dependence of Cole-Cole plots for the nonlinear susceptibilities $C_{2}^{\prime \prime}\left(C_{2}^{\prime}\right)$, given by Eqs. (12)-(14), on the parameter of the rotational diffusion anisotropy $\xi$ for some values of the angle $\Theta$ between permanent dipole moment and the symmetry axis of the molecule. 


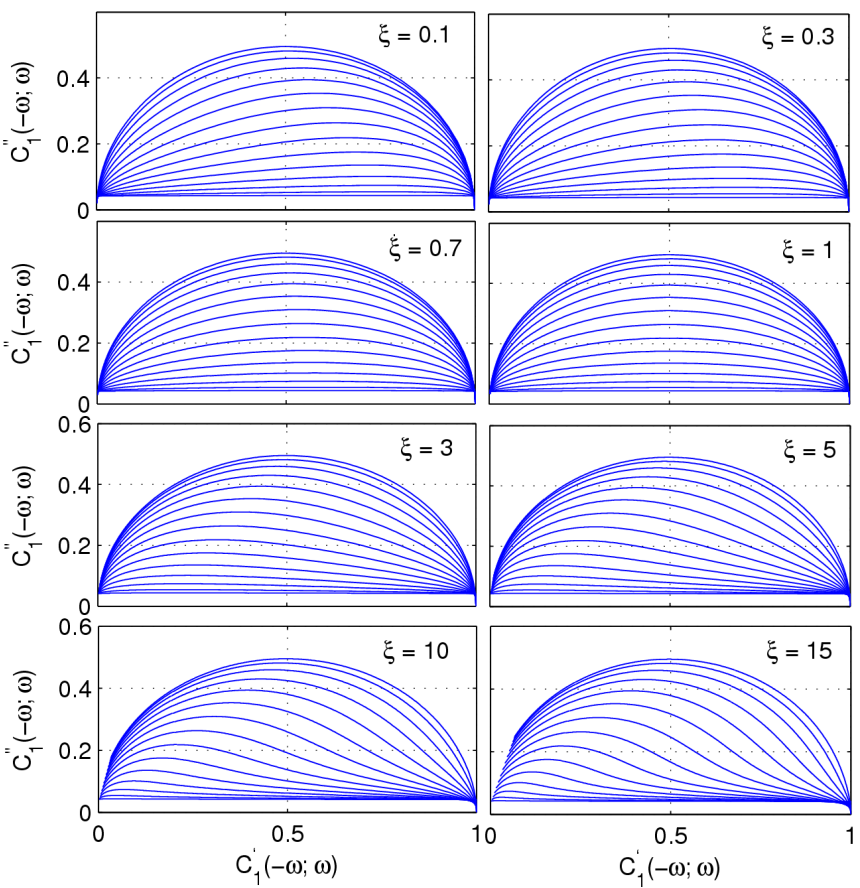

Fig. 7. Cole-Cole plots for the normalized linear electric susceptibilities $C_{1}^{\prime}(-\omega ; \omega)$ and $C_{1}^{\prime \prime}(\omega ; \omega)$ for selected values of the parameter $\xi$ and for different angles $\Theta$.

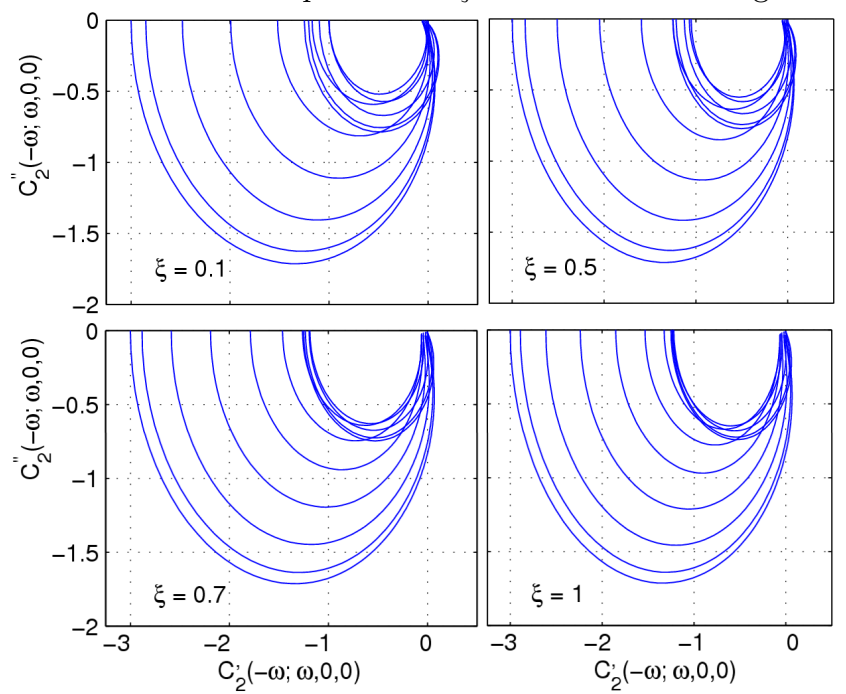

Fig. 8. Cole-Cole plots for the normalized nonlinear electric susceptibilities $C_{2}^{\prime}(-\omega ; \omega, 0,0)$ and $C_{2}^{\prime \prime}(-\omega ; \omega, 0,0)$ for rod-like molecules $(\xi=0.1 ; 0.5 ; 0.7)$ and for spherical-top molecule, $\xi=1$, for different values of the parameter $\xi$ and for different $\Theta$. 


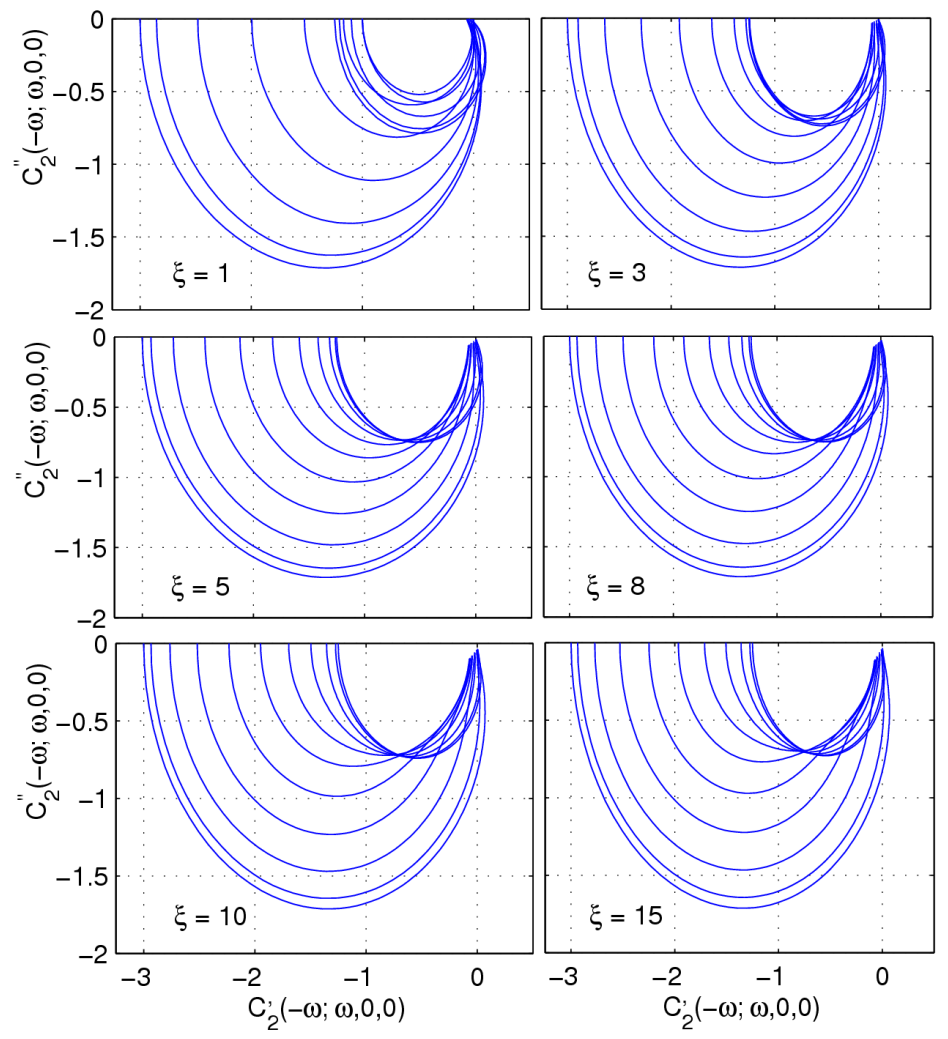

Fig. 9. Cole-Cole plots for the normalized nonlinear electric susceptibilities $C_{2}^{\prime}(-\omega ; \omega, 0,0)$ and $C_{2}^{\prime \prime}(-\omega ; \omega, 0,0)$ for disk-like molecules $(\xi>1)$ for selected values of the parameter $\xi$ and for different $\Theta$.

Figure 4 presents the Cole-Cole plots for the normalized nonlinear susceptibilities $C_{2}^{\prime}(-\omega ; \omega, 0,0)$ and $C_{2}^{\prime \prime}(-\omega ; \omega, 0,0)$ given by Eqs. (12)-(14), versus angle $\Theta$ for some values of the parameter of the rotational diffusion anisotropy $\xi$, whereas Fig. 5 shows the projections of the Cole-Cole plots $C_{2}^{\prime \prime}\left(C_{2}^{\prime}\right)$ onto the plane $\left(C^{\prime}, \Theta\right)$, for selected values of $\xi$.

In the case of nonlinear relaxation, according to Eqs. (13), (14), the resulting formulae are more complicated, of course. If the dipole moment lies in the $z$ molecular direction, $\Theta=0$, and only the terms with $a_{10}^{z z z}(\omega), b_{10}^{z z}(\omega)$, independent of $\xi$, contribute to the Langevin relaxation. Therefore, similarly as in the linear case, the spherical Smoluchowski-Debye equation is a good approximation. When $\Theta=90^{\circ}$ this term does not contribute to the susceptibilities, which now depend on the terms with $a_{11}^{x x x}(\omega, \xi), b_{11}^{x x x}(\omega, \xi)$, only. For the other values of $\Theta$ the "mixed terms", with $a_{10}^{x x z}(\omega, \xi), a_{11}^{x z z}(\omega, \xi), b_{10}^{x x z}(\omega, \xi), b_{11}^{x z z}(\omega, \xi)$, contribute and the influence of anisotropy of the diffusion tensor on the nonlinear Cole-Cole diagrams is important. 
The next Fig. 6 shows the Cole-Cole plots of the nonlinear susceptibilities $C_{2}^{\prime \prime}(-\omega ; \omega, 0,0)$ and $C_{2}^{\prime}(-\omega ; \omega, 0,0)$ given by Eqs. (12)-(14) versus the parameter $\xi$, for selected values of the angle $\Theta$ between the permanent dipole moment and the symmetry axis of the molecule, respectively, equal: $\pi / 8, \pi / 4,3 \pi / 8$.

Two-dimensional Cole-Cole plots for the normalized linear electric susceptibilities $C_{1}^{\prime}(\omega, \xi, \Theta)$ and $C_{1}^{\prime \prime}(\omega, \xi, \Theta)$, given by Eqs. (7), are presented in Fig. 7, for selected values of the parameter $\xi$ and for different polar angles $\Theta$.

Finally the Cole-Cole plots for the normalized nonlinear electric susceptibilities $C_{2}^{\prime}(-\omega ; \omega, 0,0)$ and $C_{2}^{\prime \prime}(-\omega ; \omega, 0,0)$, given by Eqs. (13), (14), are given in Fig. 8:

- for rod-like molecules $(\xi=0.1 ; 0.5 ; 0.7)$ and for spherical-top molecule, $\xi=1$, for different $\xi$ and for different values of the polar angle $\Theta$,

— and in Fig. 9, for disk-like molecules $(\xi>1)$, for selected $\xi$ and $\Theta$.

\section{References}

[1] W. Alexiewicz, K. Grygiel, Acta Phys. Pol. A 114, 667 (2008).

[2] S. Kielich, Nonlinear Molecular Optics, PWN, Warsaw 1977 (in Polish); Nauka, Moscow 1981 (in Russian).

[3] B. Kasprowicz-Kielich, S. Kielich, Adv. Mol. Relaxation Processes 7, 275 (1975).

[4] W. Alexiewicz, B. Kasprowicz-Kielich, Adv. Chem. Phys. 85, 1 (1993).

[5] W. Alexiewicz, Mol. Phys. 83, 245 (1994).

[6] W. Alexiewicz, Acta Phys. Pol. B 31, 1051 (2000).

[7] W. Alexiewicz, Chem. Phys. Lett. 320, 582 (2000).

[8] P. Debye, Polare Molekeln, Hirzel, Leipzig, 1929; Polar Molecules, Dover, New York 1945.

[9] H. Watanabe, A. Morita, Adv. Chem. Phys. 56, 55 (1984).

[10] A. Morita, H. Watanabe, J. Chem. Phys. 77, 1193 (1982).

[11] W.T. Coffey, Yu.P. Kalmykov, J.T. Waldron, The Langevin Equation With Applications in Physics, Chemistry and Electrical Engineering, World Sci., Singapore 1996.

[12] J.L. Dejardin, Dynamic Kerr Effect. The Use and Limits of the Smoluchowski Equation and Nonlinear Inertial Responses, World Sci., Singapore 1995.

[13] J.L. Dejardin, Yu.P. Kalmykov, J. Chem. Phys. 107, 508 (1997); 108, 3081 (1998).

[14] Y.P. Kalmykov, Phys. Rev. E 65, 021101 (2001).

[15] A. Chełkowski, Dielectric Physics, PWN, Warsaw, Elsevier, Amsterdam 1980.

[16] W.T. Coffey, J. Mol. Liquids 114, 5 (2004).

[17] J. Jadżyn, L. Hellemans, Acta Phys. Pol. A 67, 1093 (1985).

[18] J. Jadżyn, J.P. Parneix, C. Legrand, R. Njeumo, R. Dąbrowski, Acta Phys. Pol. A 71, 53 (1987).

[19] P. Kędziora, J. Jadżyn, K. De Smet, L. Hellemans, Chem. Phys. Lett. 289, 541 (1998). 
[20] J. Jadżyn, P. Kȩdziora, L. Hellemans, K. De Smet, Chem. Phys. Lett. 302, 337 (1999).

[21] J. Jadżyn, P. Kȩdziora, L. Hellemans, Phys. Lett. A 251, 49 (1991). 